\title{
Medical Evaluation of Marker Controlled Watershed Technique for Detecting Brain Tumors
}

\author{
Navdeep Kumar \\ Department of Computer Science-SVIET \\ Banur(India)
}

\author{
Sugandha Arora \\ Department of Computer Science-SVIET \\ Banur(India)
}

\begin{abstract}
Image Segmentation has a vital role in the field of Medical Science especially in the area of cancer research for detecting tumors. Proper diagnosis and effective treatment is only possible if we have an efficient segmenting technique. In this paper we present a sound segmentation technique named as marker controlled watershed technique which is based on repeated morphological image dilation and erosion operations to carry out segmentation. After segmenting the desired area we evaluate this technique for its accuracy and performance.
\end{abstract}

\section{Keywords}

Watershed Method, ROI, Segmentation, Brain Tumor, Image Dilation, Image Erosion.

\section{INTRODUCTION}

One of most challenging task in the medical science is the detection of cancerous tumor in early stage. Difficulty arises due to overlapping of normal with abnormal tissue in grey level of medical images [2]. So it is mandatory for the surgeon to accurately delineate the boundaries of abnormal tissue from healthy tissues so as to remove it without damaging the healthy parts. He can perform his job efficiently if he has accurate dimensions of the part to be separated. As brain tumor has deadly effect in later stages on human body, it is necessary to have high accuracy requirement in diagnosis at early stage. Medical imaging has become an indispensable tool in today's medical treatment. A large amount of data and information about a disease process is acquired through various image acquisition techniques such as Medical Resonance Imaging (MRI), Computer Tomography (CT) etc [4]. Segmenting a medical image is useful step for detecting and locating various types of irregular structures in human body such as bone fractures and tumors. In other words medical image segmentation plays a vital role in diagnosing and treatment of various hazardous diseases like cancer. Extracting a desired object area from an image using thresholding [5] method is very basic in which Region of Interest (ROI) is extracted using a predefined threshold value. The intensity values above the threshold value are delineated into one region and below the threshold make another separate region. In this method it is very difficult to mark an appropriate threshold. So it is not suitable for complex medical images. Another basic technique is Geodesic Active Contour model proposed by Chan and Vese [6]. This technique is based on curve evolution and minimizing the energy function. Curve evolution stops at the boundaries of the objects. Since this technique does not use gradient edges, it is not suitable for detecting very fine edges as in case of medical images where boundary between objects is very fine and sometime may be overlapping. Li and Lui proposed another segment method based on Gradient Vector flow [7] to overcome the problem of passing the contour into the week object boundary. This method gained popularity because of its capability of automatically initialization and splitting of curve at different object boundaries. But as the number of objects increase in the image it tends to go down in computation speed. The most popular technique in contour evolution is Distance Regularized Level Set (DRLS) [8] in which distance regularize term is used which defined with the signed distance function for curve evolution and eliminates the reinitialization and thereby reduces the induced numerical errors. This method suffers greatly when objects being detected are having irregular shape.

\section{WATERSHED TRANSFORM}

The term watershed is derived from the area where if water is made to fall on it at a uniform rate would occupy the region minimum. The basic concept of watershed is based on visualizing a gray level image into its topographic representation, which includes three basic notions: minima, catchment basins and watershed lines. Fig. 1.2 illustrates the meanings of these definitions. In the image of Fig. 1.2(a), if we imagine the bright areas have "high" altitudes and dark areas have "low" altitudes, then it might look like the topographic surface illustrated by Fig. 1.2(b). In this surface, it is natural to consider three types of points: (1) points belonging to the different minima; (2) points at which water would fall with certainty to a single minimum; and (3) points at which water would be equally likely to fall to more than one minimum. The first type of points forms different minima of the topographic surface. The second type points which construct a gradient interior region is called catchment basin. The third type of points form crest lines dividing different catchment basins, which is termed by watershed lines.

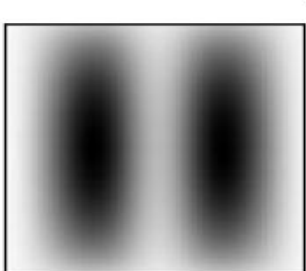

(a)

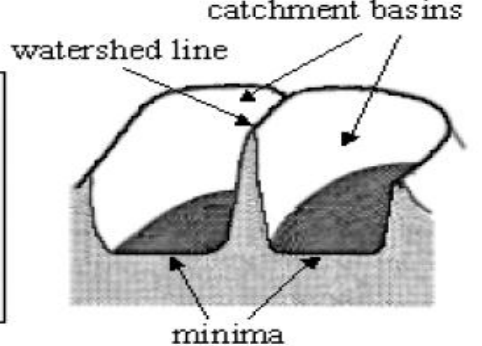

(b)
Fig 1: The system conceptual diagram

\section{OVERVIEW OF PROPOSED WORK}

\subsection{Segmentation}

In our method we propose a new watershed technique in which segmentation is achieved using repeated morphological operations. In this technique we first take the image gradient and morphological operations are applied on this image 
gradient. We first apply image erosion followed by image dilation. After image dilation we got a number of image minimum. Here the problem of oversegmentation may occur which is undesired. To overcome this drawback we mark the foreground objects and background objects by opening followed by closing by reconstruction. After this operation we got limited number of region minimum which is a desired outcome. Now the final result of this segmentation method is achieved using appropriate structuring element which separate the region of interest from rest of the image.

\subsection{Feature Extraction}

Before evaluating the effectiveness of the marker controlled watershed transform it is required to extract the meaningful features from segmented areas. There are so many features which can be extracted from a given image but we are concerned only texture features. To extract the texture feature we used Grey Level Co-occurrence Matrix (GLCM). Using GLCM we extracted 22 Texture features. From these extracted features we used 5 features to evaluate the segmented area of tumor.

\section{RESULTS}

We have used MATLAB 7.6 to implement our technique. We took three sample images of data set images of 30 images from an open source database of brain tumor MRI images. From this image dataset we have also acquired ground truth of brain tumor. To evaluate the effectiveness of our proposed method we compare our results with ground truths. Our results have been shown in the following figure
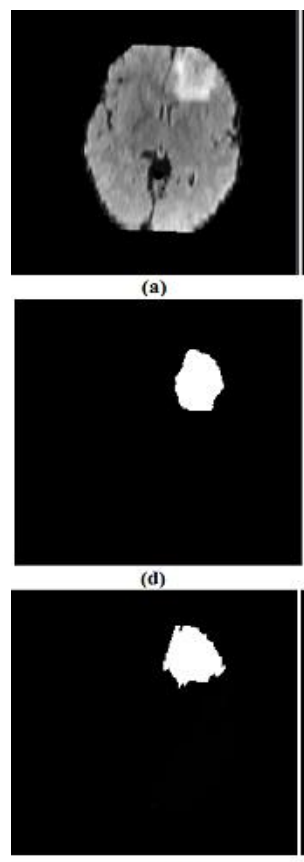

(g)
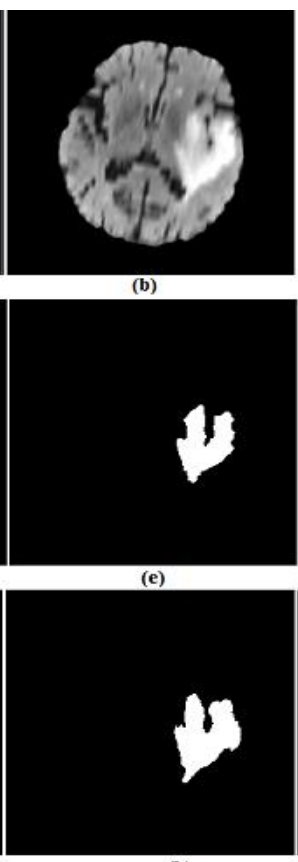

(h)
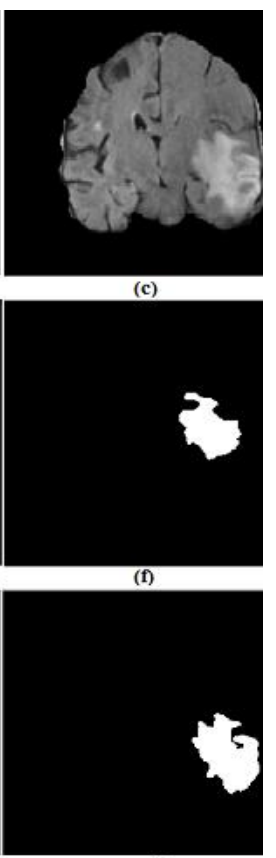

(i)
Fig 2: Comparative results of the proposed method. (a-bc) Original images. (d-e-f) Ground truth. (g-h-i) segmented by proposed method.

After segmenting the tumor region with our method, we extracted some useful texture features of the segmented area. Although there are so many feature which can be extracted from these images, our method required only five features for evaluation. The tables of these features are shown below:
Table 4.a: Comparison of Extracted Features of Sample image (a)

\begin{tabular}{|l|l|l|}
\hline \multirow{2}{*}{$\begin{array}{l}\text { Extracted } \\
\text { Features from } \\
\text { images }\end{array}$} & $\begin{array}{l}\text { Ground } \\
\text { Truth Region }\end{array}$ & $\begin{array}{l}\text { Segmented Region } \\
\text { using Proposed } \\
\text { Method }\end{array}$ \\
\cline { 2 - 3 } Energy & $9.3322 \mathrm{e}-001$ & $9.1912 \mathrm{e}-001$ \\
\hline Entropy & $1.6177 \mathrm{e}-001$ & $1.9771 \mathrm{e}-001$ \\
\hline Autocorrelation & $1.0977 \mathrm{e}+000$ & $1.1108 \mathrm{e}+000$ \\
\hline Variance & $6.4517 \mathrm{e}-001$ & $6.5301 \mathrm{e}-001$ \\
\hline Homogeneity & $9.9858 \mathrm{e}-001$ & $9.9626 \mathrm{e}-001$ \\
\hline
\end{tabular}

Table 4.b: Comparison of Extracted Features of Sample image (b)

\begin{tabular}{|c|c|c|}
\hline \multirow[b]{2}{*}{$\begin{array}{l}\text { Extracted } \\
\text { Features from } \\
\text { images }\end{array}$} & \multicolumn{2}{|c|}{ Image (b) } \\
\hline & $\begin{array}{l}\text { Ground Truth } \\
\text { Region }\end{array}$ & $\begin{array}{l}\text { Segmented Region } \\
\text { using Proposed } \\
\text { Method }\end{array}$ \\
\hline Energy & $9.1609 \mathrm{e}-001$ & $8.8454 \mathrm{e}-001$ \\
\hline Entropy & $2.0425 \mathrm{e}-001$ & $2.6536 \mathrm{e}-001$ \\
\hline Autocorrelation & $1.1147 \mathrm{e}+000$ & $1.1580 \mathrm{e}+000$ \\
\hline Variance & $6.5646 \mathrm{e}-001$ & $6.8939 \mathrm{e}-001$ \\
\hline Homogeneity & $9.9602 \mathrm{e}-001$ & $9.9389 \mathrm{e}-001$ \\
\hline
\end{tabular}

Table 4.c: Comparison of Extracted Features of Sample image (c)

\begin{tabular}{|l|l|l|}
\hline \multirow{2}{*}{$\begin{array}{l}\text { Extracted } \\
\text { Features from } \\
\text { images }\end{array}$} & $\begin{array}{l}|c| \\
\text { Ground Truth } \\
\text { Region }\end{array}$ & $\begin{array}{l}\text { Segmented Region } \\
\text { using Proposed } \\
\text { Method }\end{array}$ \\
\hline Energy & $9.8392 \mathrm{e}-001$ & $8.1964 \mathrm{e}-001$ \\
\hline Entropy & $3.0744 \mathrm{e}-001$ & $3.9097 \mathrm{e}-001$ \\
\hline Autocorrelation & $1.0223 \mathrm{e}+000$ & $1.2456 \mathrm{e}+000$ \\
\hline Variance & $6.8150 \mathrm{e}-001$ & $7.7222 \mathrm{e}-001$ \\
\hline Homogeneity & $9.9950 \mathrm{e}-001$ & $9.8803 \mathrm{e}-001$ \\
\hline
\end{tabular}

To measure the accuracy of our method we compare our results with ground truths of the brain tumor and we have found that our method have $85 \%$ accuracy. We also compare our proposed method with other segmentation methods and 
found that our method has less computation time than other segmentation methods.

\section{DISCUSSIONS AND CONCLISION}

As we have implemented our segmentation method. It can be easily predicted that our segmentation method is semiautomatic and required no human intervention as in case of other segmented techniques. Moreover our proposed method has better accuracy and less computation time. The results of our method would be more accurate if the difference of intensity levels of tumor and non tumor region is high. Our method can even detect the segmented area of low intensity images as well if proper parameters are set according to the image being segmented.

As we know that Watershed Techniques has the ability to detect the continuous boundary of the region of interest, it can be best suited for those types of applications where high accuracy and precision is needed. Detection of tumor in the area of cancer research is best suited area of application where watershed segmentation can be applied efficiently.

\section{REFERENCES}

[1] Marc Macenko, Mehmet Celenk, Limin Ma, Lesion Detection Using Morphological Watershed and Modal based Inverse filtering, The $18^{\text {th }}$ International Conference on Pattern Recognition 2006 IEEE.

[2] Saif D. Salman, Ahmad A. Behrani, Segmentation of tumor tissue in grey medical images using watershed transform methods, International Journal of Advancement in Computing Technology. October 2010

[3] Jun Liu, Jianxun Chen, Xiaoming Liu, Lei Chun, Jinshan tang,Youping Deng, Mass Segmentation using a combined method of cancer Detection, BMC System Biology 2011
[4] D. Jayadevappa, S. Srinivas Kuamr, D.S. Murthy, A Hybrid Segmentation Model based on Watershed and Gradient Vector flow of the detection of tumor, International Journal of Signal Processing, Image processing and Pattern Recognition 2009.

[5] Mohammad N. Nobi, Faruk Chowdhury, Faysal Muhammad, Muhammad A. Yusuf, A New Medical Image Segmentation Technique Based on variatioanl Level Set Method, International Journal of Computer and Electrical Engineering October 2011

[6] Tony F. Chan, Luminita A. Vese, Active Contours Without Edges, IEEE Transactions on Image Processing 2001

[7] Chunming Li ,Jundong Liu, Segmentation of Edge Preserving Gradient Vector Flow: An Approach TowardAutomatically Initializing and Splitting of Snakes. Computer Society Conference on Computer Vision and Pattern Recognition IEEE 2005

[8] Chunming Li, Chenyang, Xu Distance Regularized Level Set Evolution and Its Application to Image Segmentation, IEEE Transaction on Image Processing, 2010.

[9] Dr. H.B. Kekre, Tanuja K. Sarode, Saylee M. Gharge, Tumor Detection in Mammography Images using Vector Quantization Technique, International Journal of Intelligent Information Technology Application 20009

[10] H.P.Ng, S.H.Ong, K.W.C. Foong, P.S. Goh, W.L. Nowinski,Medical Image segmentation using $k$ means clustering and improved watershed algorithm .IEEE 2006 